\title{
Biorthogonal Nonunifrom B-spline Wavelets with Small Supports
}

\author{
Pan Rijing ${ }^{1,3}$,Yao Zhiqing ${ }^{2,3}$ \\ 1. college of mathematics and computer science, fujian normal university, Fuzhou, Fujian 350007, P R China \\ 2. Faculty of Software, Fujian Normal University, Fuzhou, Fujian 350007, P R China \\ 3. Key Lab of Network Security and Cryptology, Fujian Normal University, Fuzhou, Fujian 350007, P R China
}

\begin{abstract}
A kind of biorthogonal nonuniform B-spline wavelets with small supports is constructed. And the performances of the wavelets are analyzed. In additional to having small supports, the proposed wavelets are simple and efficient in computation, possess good approximation property and are flexible for the application in multiresolution modeling of NURBS curves and surfaces.
\end{abstract}

Keywords-B-spline wavelets, nonuniform B-spline, biorthogonal, multiresolution, support

\section{INTRODUCTION}

Multiresolution modeling of curves and surfaces is an important area in CAGD. B-spline wavelets are the bases of multiresolution representation of parametric curves and surfaces. Unifrom or quasiuniform B-spline wavelets have been applied in the modeling of curves and surfaces earlier [1]. But they cannot be applied directly to nonuniform Bspline based NURBS curves and surfaces. Semiorthogonal nonuniform B-spline wavelets are suitable for NURBS [2], but they are complex in computation. Biorthogonal nonuniform B-spline wavelets are simpler in computation usually. There are a few research results in the aspect [3-5]. The Biorthogonal nonuniform B-spline wavelets proposed in [5] are based on discrete norm $l_{2}$ which are efficient in computation, good in approximation and flexible in application. But their supports are not always small when the knots with multiplicities greater than one are inserted. Small supports of wavelets provide greater flexibility for the multiresolution modeling of curves and surfaces. This paper improves the results in [5], and proposes a king of biorthogonal nonuniform B-spline wavelets which possess smaller supports and retain the strong points of the wavelets in $[5]$.

\section{BIORTHOGONAL NONUNIFORM B-SPLINE}

\section{WAVELETS}

Let $\boldsymbol{T}_{0} \subset \boldsymbol{T}_{1} \subset \cdots$ be a nested sequence of nonuniform knot vectors, where $\boldsymbol{T}_{i}=\left\{t_{i, 0}, t_{i, 1}, \cdots, t_{i, n_{i}+k}\right\}, i=0,1, \cdots$ satisfy the following conditions:

$$
\begin{aligned}
& a=t_{i, 0}=\cdots=t_{i, k-1}<t_{i, k} \leq t_{i, k+1} \leq \cdots \leq t_{i, n_{i}}<t_{i, n_{i}+1}=\cdots=t_{i, n_{i}+k}=b, \\
& t_{i, j}<t_{i, j+k}, n_{i} \geq k-1,
\end{aligned}
$$

$\left\{N_{i, j, k}(t)\right\}_{j=0}^{n_{i}}$ be the normalized B-spline basis of order $k$ determined by knot vector $\boldsymbol{T}_{\boldsymbol{i}}$, and $\boldsymbol{V}_{i}$ be the polynomial spline space of degree $k-1$ spanned by $\left\{N_{i, j, k}(t)\right\}_{j=0}^{h_{i}}$. Then $\boldsymbol{V}_{0} \subset \boldsymbol{V}_{1} \subset \cdots$. Suppose that $\boldsymbol{W}_{i}$ is a complement space of $\boldsymbol{V}_{i}$ in $\boldsymbol{V}_{i+1}$. Then a basis $\left\{\Psi_{i, j}(t)\right\}_{j=1}^{m_{i}}$ of $\boldsymbol{W}_{i}$ constitutes a set of nonuniform B-spline wavelets, where $m_{i}+n_{i}=n_{i+1} \cdot \boldsymbol{V}_{i}$ and $\boldsymbol{W}_{i}$ are called scale and wavelet spaces respectively. Let $\boldsymbol{N}_{i, k}=\left\lfloor N_{i, 0, k} N_{i, 1, k} \cdots N_{i, n_{i}, k}\right\rfloor$ and $\boldsymbol{\Psi}_{i}=\left[\Psi_{i, 1} \Psi_{i, 2} \cdots \Psi_{i, m_{i}}\right]$. Then there exist matrices $\boldsymbol{P}_{i}$ of order $\left(n_{i+1}+1\right) \times\left(n_{i}+1\right)$ and $\boldsymbol{Q}_{i}$ of order $\left(n_{i+1}+1\right) \times m_{i}$ such that

$$
\left\lfloor\begin{array}{ll}
\boldsymbol{N}_{i, k} & \boldsymbol{\Psi}_{i}
\end{array}\right]=\boldsymbol{N}_{i+1, k}\left[\begin{array}{ll}
\boldsymbol{P}_{i} & \boldsymbol{Q}_{i}
\end{array}\right]
$$

where $\boldsymbol{P}_{i}$ and $\boldsymbol{Q}_{i}$ are called the reconstruction matrices of the B-spline wavelets. Let $\left[\begin{array}{l}\boldsymbol{A}_{i} \\ \boldsymbol{B}_{i}\end{array}\right]=\left[\begin{array}{ll}\boldsymbol{P}_{i} & \boldsymbol{Q}_{i}\end{array}\right]^{-1}$. Then $\boldsymbol{A}_{i}$ of order $\left(n_{i}+1\right) \times\left(n_{i+1}+1\right)$ and $\boldsymbol{B}_{i}$ of order $m_{i} \times\left(n_{i+1}+1\right)$ are called the decomposition matrices of the B-spline wavelets. The construction of B-spline wavelets means construction matrices $\boldsymbol{P}_{i}, \boldsymbol{Q}_{i}, \boldsymbol{A}_{i}$ and $\boldsymbol{B}_{i}$.

Reconstruction matrix $\boldsymbol{P}_{i}$ is a knot insertion matrix for every kind of B-spline wavelets. It can be computed by Oslo Algorithm [6] or the recursive algorithm [7]. But for different kinds of B-spline wavelets,

their reconstruction matrices $\boldsymbol{Q}$ 's are different. And the constructions of decomposition matrices depend on that of reconstruction matrices. Therefore the key for constructing B-spline wavelets is constructing $\boldsymbol{Q}$. In order to construct biorthogonal B-spline wavelets, though the condition for $\boldsymbol{Q}_{i}$ is only that $\left[\begin{array}{ll}\boldsymbol{P}_{i} & \boldsymbol{Q}_{i}\end{array}\right]$ is a nonsingular matrix theoretically, there are more requirements usually according to the demands of multiresolution modeling of curves and surfaces. The performances required for wavelets include: (i) simple and efficient computation; (ii) good approximation property; (iii) small support; (iv) flexible in applications. Approximation property insures that the lower resolution curves and surfaces approximate the higher resolution ones well. Wavelets with smaller supports make that the decomposed wavelet details possess better local property.

Knot vector $\boldsymbol{T}_{i+1}$ of higher resolution is constructed by inserting $m_{i}$ knots into knot vector $\boldsymbol{T}_{i}$ of lower resolution. 
One new knot corresponds to one wavelet. $\boldsymbol{Q}_{i}$ is a band matrix. According to Eq. (1) and the local support property of B-spline, the support of a wavelet is determined by the bandwidth (the number of nonzero elements) of the corresponding column in $\boldsymbol{Q}_{i}$. And one new knot $t_{i+1, j}$ only affects $\quad k+1 \quad$ B-spline base functions $N_{i+1, j-k+1, k}(t), N_{i+1, j-k+2, k}(t), \cdots, N_{i+1, j, k}(t)$. So it is enough and suitable using these $k+1$ base functions to represent the wavelet corresponding to the new knot $t_{i+1, j}$. This implies that it is feasible and reasonable the bandwidth of the corresponding column in $\boldsymbol{Q}_{i}$ is $k+1$. Therefore from the view of application, the minimum column bandwidth of $\boldsymbol{Q}_{i}$ is $k+1$.

\section{NEW B-SPLINE WAVELETS WITH SMALL SUPPORTS}

\section{A. Construction Of New Wavelets}

Denote the column bandwidth of a band matrix $\boldsymbol{A}$ by $\mathrm{CW}(\boldsymbol{A})$, that is $\mathrm{CW}(\boldsymbol{A})=$ the maximal number of nonzero elements in a column of matrix $\boldsymbol{A}$.

The biorthogonal nonuniform B-spline wavelets given in [5] (called Pan wavelets below) possess properties (i), (ii) and (iv) proposed in section 2. Let $\overline{\boldsymbol{Q}}_{i}$ be the reconstruction matrix of Pan wavelets. In general cases $\mathrm{CW}\left(\overline{\boldsymbol{Q}}_{i}\right)=k+1$, but it is possible that $\mathrm{CW}\left(\overline{\boldsymbol{Q}}_{i}\right)>k+1$ when knots with multiplicities greater than one are inserted. Based on Pan wavelets we construct new B-spline wavelets below. The new wavelets possess smaller supports such that $\mathrm{CW}\left(\overline{\boldsymbol{Q}}_{i}\right)=k+1$ and maintain the good properties (i), (ii) and (iv) as that of Pan wavelets.

Firstly we analyze the distribution of nonzero elements of $\overline{\boldsymbol{Q}}_{i}$. Let $t_{i+1, j} \in \boldsymbol{T}_{i+1}$ be a new knot corresponding to $\boldsymbol{T}_{i}$ and its insertion multiplicity be $\alpha_{i+1}(j)$. Then according to the construction formula of $\overline{\boldsymbol{Q}}_{i}$ in [5], the nonzero elements in $\alpha_{i+1}(j)$ columns of $\overline{\boldsymbol{Q}}_{i}$ corresponding to $t_{i+1, j}$ distribute as follows:

$$
\begin{aligned}
& \tau_{i+1}(j)=\text { Themultiplityjof } t_{i+1, j} \text { in } \boldsymbol{T}_{i}, \quad l_{i+1}(j)=\min \left\{\left.\right|_{i+1, s}=t_{i+1, j}, 0 \leq s \leq n_{i+1}\right\} \\
& \beta_{i+1}(j)=l_{i+1}(j)-k+\tau_{i+1}(j)+\alpha_{i+1}(j)-1 .
\end{aligned}
$$

From Eq. (2) we know that the bandwidth of the corresponding column in $\overline{\boldsymbol{Q}}_{i}$ is $k+1$ when $\alpha_{i+1}(j)=1$, the bandwidths of $\alpha_{i+1}(j)$ corresponding columns in $\overline{\boldsymbol{Q}}_{i}$ are greater than $k+1$ possibly when $\alpha_{i+1}(j)>1$, and in the worst case that reach $2 k$ when $\alpha_{i+1}(j)=k$.

Define the discrete inner product of space $\boldsymbol{V}_{i}$ as $<h_{1}, h_{2}>=\boldsymbol{v}_{1}^{\mathrm{T}} \boldsymbol{v}_{2}$, where $h_{1}=\boldsymbol{N}_{i, k} \boldsymbol{v}_{1}, h_{2}=\boldsymbol{N}_{i, k} \boldsymbol{v}_{2}$. Pan wavelets make that $\boldsymbol{W}_{i}$ and $\boldsymbol{V}_{i}$ are orthogonal based on this discrete inner product. So the reconstruction matrices satisfy the following discrete orthogonal condition:

$$
\boldsymbol{P}_{i}^{\mathrm{T}} \overline{\boldsymbol{Q}}_{i}=\mathbf{0} .
$$

Obviously, Eq. (4) is still true if we execute any column transformation to $\overline{\boldsymbol{Q}}_{i}$, that is $\boldsymbol{P}_{i}^{\mathrm{T}} \widetilde{\boldsymbol{Q}}_{i}=\boldsymbol{0}$ for $\widetilde{\boldsymbol{Q}}_{i}=\overline{\boldsymbol{Q}}_{i} \boldsymbol{D}$ where $\boldsymbol{D}$ is an arbitrary nonsingular matrix of order $m_{i}$. This means that if new wavelets are defined with $\widetilde{\boldsymbol{Q}}_{i}$ as wavelet reconstruction matrix, the new wavelets possess the same approximation property as Pan wavelets. But from Eq. (2) we know that executing column transformation to $\overline{\boldsymbol{Q}}_{i}$ cannot reduce the maximum column bandwidth to $k+1$ if $\operatorname{CW}\left(\overline{\boldsymbol{Q}}_{i}\right)>k+1$. It is observed that if we execute proper row transformation to $\overline{\boldsymbol{Q}}_{i}$ first, then reducing the maximum column bandwidth to $k+1$ through column transformation can be realized.

The method for the construction of new wavelets is based on the above analysis. Firstly define a row transformation matrix of order $n_{i+1}$ as follows:

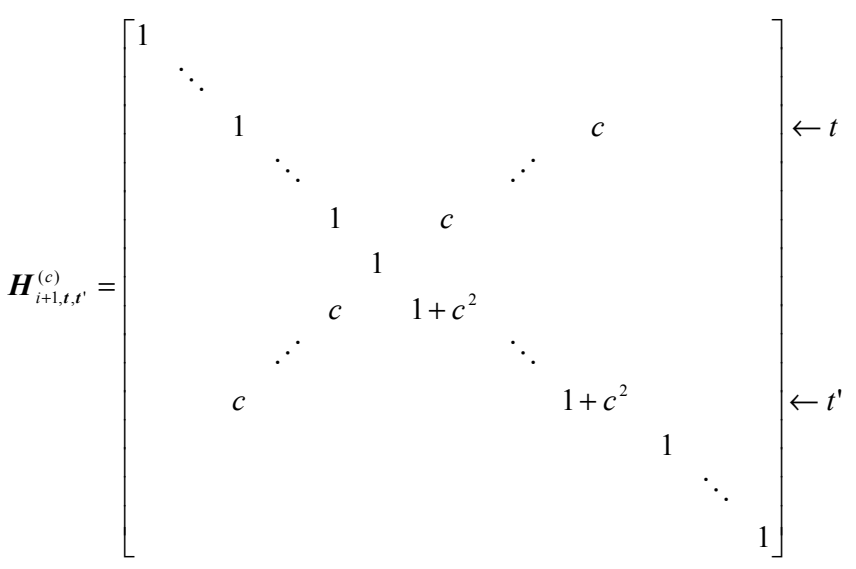

where $t^{\prime}-t$ is oven, $c$ is a real number, $0<c<1$, the other elements are all 0 's. For the case when $t^{\prime}-t$ is odd, matrix $\boldsymbol{H}_{i+1, t, t^{\prime}}^{(c)}$ is defined similarly. Suppose that $t_{i+1, j_{1}}, t_{i+1, j_{2}}, \cdots, t_{i+1, j_{r}} \in \boldsymbol{T}_{i+1}$ are all new knots corresponding to $\boldsymbol{T}_{i}$ with the insertion multiplicities $\alpha_{i+1}\left(j_{s}\right)>1$. Let

where \# denotes a nonzero element, 
$\boldsymbol{H}_{i+1}^{(c)}=\prod_{s=1}^{r} \boldsymbol{H}_{i+1, \gamma_{i+1}\left(j_{s}\right), \beta_{i+1}\left(j_{s}\right)}^{(c)}$, where $\gamma_{i+1}\left(j_{s}\right)=\beta_{i+1}\left(j_{s}\right)-\alpha_{i+1}\left(j_{s}\right)+1$, $\beta_{i+1}\left(j_{s}\right)$ is defined by Eq. (3). Execute the row transformation defined by $\boldsymbol{H}_{i+1}^{(c)}$ to $\overline{\boldsymbol{Q}}_{i}$ and denote the result matrix as $\hat{\boldsymbol{Q}}_{i}=\boldsymbol{H}_{i+1}^{(c)} \overline{\boldsymbol{Q}}_{i}$. According to the function of row transformation matrix $\boldsymbol{H}_{i+1}^{(c)}$ and the structure of $\overline{\boldsymbol{Q}}_{i}$ showed by Eq. (2), then $\hat{\boldsymbol{Q}}_{\boldsymbol{i}}$ can be eliminate elements through column transformation to make its maximal column bandwidth not greater than $k+1$. Suppose the corresponding column transformation matrix is $\boldsymbol{L}_{\boldsymbol{i}}$ and let $\boldsymbol{Q}_{i}=\hat{\boldsymbol{Q}}_{i} \boldsymbol{L}_{i}=\boldsymbol{H}_{i+1}^{(c)} \overline{\boldsymbol{Q}}_{\boldsymbol{i}} \boldsymbol{L}_{\boldsymbol{i}}$. Then $\mathrm{CW}\left(\boldsymbol{Q}_{\boldsymbol{i}}\right)=k+1$. Since according to Eq. (4) $\boldsymbol{Q}_{i}$ satisfies

$$
\boldsymbol{P}_{i}^{\mathrm{T}} \boldsymbol{H}_{\boldsymbol{i}+1}^{(c)-1} \boldsymbol{Q}_{\boldsymbol{i}}=\mathbf{0},
$$

$\left[\begin{array}{ll}\boldsymbol{P}_{i} & \boldsymbol{Q}_{i}\end{array}\right]$ is a nonsingular matrix. This implies that $\boldsymbol{Q}_{i}$ can be used as wavelet reconstruction matrix. Now we have obtained the new biorthogonal nonuniform B-spline wavelets with small supports $\left(\mathrm{CW}\left(\boldsymbol{Q}_{i}\right)=k+1\right)$ defined by $\boldsymbol{P}_{i}$ and $\boldsymbol{Q}_{i}$ which are called new wavelets below.

\section{B. Algorithms Of New Wavelets}

The following is the reconstruction algorithm for new wavelets.

Algorithm reconstruction

Input: level number $i$, coefficient vector of lower resolution $\boldsymbol{d}_{i}$, wavelet coefficient vector $\boldsymbol{w}_{i}$, order of B-spline $k$, knot vector $\boldsymbol{T}_{i}$ and $\boldsymbol{T}_{i+1}$.

Output: reconstruction matrices $\boldsymbol{P}_{i}$ and $\boldsymbol{Q}_{i}$, coefficient vector of higher resolution $\boldsymbol{d}_{i+1}$.

Step 1 Compute matrix $\boldsymbol{P}_{i}$ recursively by using the algorithm in [5];

Step 2 Compute matrix $\overline{\boldsymbol{Q}}_{i}$ recursively by using the algorithm in [5];

Step 3 Compute $\hat{\boldsymbol{Q}}_{\boldsymbol{i}}=\boldsymbol{H}_{\boldsymbol{i}+1}^{(c)} \overline{\boldsymbol{Q}}_{\boldsymbol{i}}$;

Step 4 Eliminate elements of $\hat{\boldsymbol{Q}}_{i}$ through column transformation, and obtain the result matrix $\boldsymbol{Q}_{i}$ with $\mathrm{CW}\left(\boldsymbol{Q}_{i}\right)=k+1$;

Step 5 Compute $\boldsymbol{d}_{i+1}=\boldsymbol{P}_{i} \boldsymbol{d}_{i}+\boldsymbol{Q}_{i} \boldsymbol{w}_{i}$.

Then we consider the decomposition computation. According to $\boldsymbol{P}_{i} \boldsymbol{A}_{i}+\boldsymbol{Q}_{i} \boldsymbol{B}_{i}=\boldsymbol{I}$ and noting that $\boldsymbol{H}_{i+1}^{(c)}$ is a symmetric matrix, we have

$$
\begin{array}{r}
\boldsymbol{A}_{\boldsymbol{i}}=\left(\boldsymbol{P}_{i}^{\mathrm{T}} \boldsymbol{H}_{i+1}^{(c)-1} \boldsymbol{P}_{\boldsymbol{i}}\right)^{-1} \boldsymbol{P}_{i}^{\mathrm{T}} \boldsymbol{H}_{\boldsymbol{i}+1}^{(c)-1}, \\
\boldsymbol{B}_{\boldsymbol{i}}=\left(\boldsymbol{Q}_{i}^{\mathrm{T}} \boldsymbol{H}_{\boldsymbol{i}+1}^{(c)-1} \boldsymbol{Q}_{\boldsymbol{i}}\right)^{-1} \boldsymbol{Q}_{i}^{\mathrm{T}} \boldsymbol{H}_{\boldsymbol{i}+1}^{(c)-1}, \\
\text { where } \boldsymbol{H}_{i+1}^{(c)-1}=\prod_{s=1}^{r} \boldsymbol{H}_{i+1, \gamma_{i+1}\left(j_{s}\right), \beta_{i+1}\left(j_{s}\right),}^{(c)-1}
\end{array}
$$

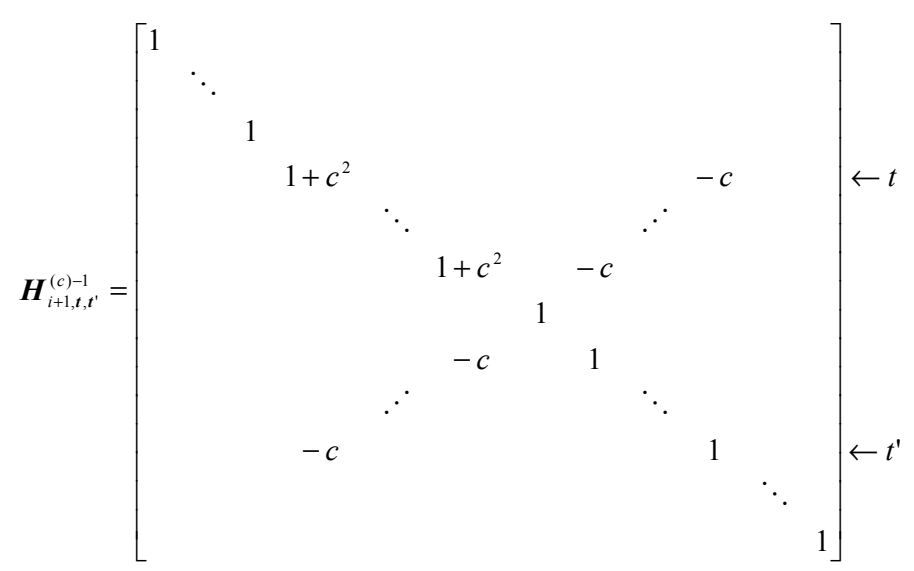

when $t^{\prime}-t$ is oven, and $\boldsymbol{H}_{i+1, t, t^{\prime}}^{(c)-1}$ is represented similarly when $t^{\prime}-t$ is odd.

Though we can compute decomposition matrices $\boldsymbol{A}_{i}$ and $\boldsymbol{B}_{i}$ according to Eq. (6) firstly, then compute coefficient vector of lower resolution $\boldsymbol{d}_{i}=\boldsymbol{A}_{i} \boldsymbol{d}_{i+1}$ and wavelet coefficient vector $\boldsymbol{w}_{i}=\boldsymbol{B}_{i} \boldsymbol{d}_{i+1}$, it will result in more expensive computation cost. The following is an algorithm to compute $\boldsymbol{d}_{\boldsymbol{i}}$ and $\boldsymbol{w}_{i}$ directly.

Algorithm decomposition

Input: level number $i$, order of B-spline $k$, coefficient vector of higher resolution $\boldsymbol{d}_{i+1}$, reconstruction matrices $\boldsymbol{P}_{i}$ and $\boldsymbol{Q}_{i}$.

Output: coefficient vector of lower resolution $\boldsymbol{d}_{i}$ and wavelet coefficient vector $\boldsymbol{w}_{i}$.

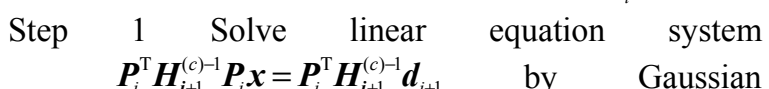
elimination to obtain $\boldsymbol{d}_{i}$;

Step 2 Solve linear equation system $\boldsymbol{Q}_{i}^{\mathrm{T}} \boldsymbol{H}_{i+1}^{(c)-1} \boldsymbol{Q}_{i} \boldsymbol{x}=\boldsymbol{Q}_{i}^{\mathrm{T}} \boldsymbol{H}_{i+1}^{(c)-1} \boldsymbol{d}_{i+1} \quad$ by Gaussian elimination to obtain $\boldsymbol{w}_{i}$.

\section{Performances Of New Wavelets}

The performances in computation efficiency, support, application and approximation property of new wavelets are analyzed below.

Firstly, except for Step 3 and 4 the reconstruction algorithm in 3.2 is the same as that for Pan wavelets [5]. And the computation time for Step 3 and 4 is $\mathrm{O}\left(k n_{i+1}\right)$ obviously since $\overline{\boldsymbol{Q}}_{i}$ is a band matrix. Because the time complexity of the reconstruction algorithm for Pan wavelets is $\mathrm{O}\left(k^{2} n_{i+1}\right)$ [5], that for new wavelets is the same. It is not difficult to analyze that the time complexity of the decomposition algorithm of new wavelets is also $\mathrm{O}\left(k^{2} n_{i+1}\right)$. So the reconstruction and decomposition computations of new wavelets both can be completed in linear time. 
Secondly, the maximal column bandwidth of reconstruction matrix $\boldsymbol{Q}_{i}$ is $k+1$. From the view of application new wavelets possess the smallest supports.

Thirdly, since new wavelets are nonuniform ones, they provide greater flexibility when applied to the MRA of NURBS curves and surfaces. They are suitable for various nested sequence of knot vectors. They can be used to construct rich multiresolution levels for various applications.

Lastly, we discuss the approximation property of new wavelets. Since $\boldsymbol{H}_{i+1}^{(c)-1}$ is a positive definite matrix, we can define a discrete inner product in space $\boldsymbol{V}_{\boldsymbol{i}+1}$ as $<h_{1}, h_{2}>_{i+1, c}=\boldsymbol{v}_{1}^{\mathrm{T}} \boldsymbol{H}_{i+1}^{(c)-1} \boldsymbol{v}_{2} \quad, \quad$ where $\quad h_{1}=\boldsymbol{N}_{i+1, k} \boldsymbol{v}_{1} \in \boldsymbol{V}_{i+1}$, $h_{2}=\boldsymbol{N}_{i+1, k} \boldsymbol{v}_{2} \in \boldsymbol{V}_{i+1}$.

Then according to Eq. (5), the scale space $\boldsymbol{V}_{i}$ and wavelet space $\boldsymbol{W}_{i}$ for new wavelets are orthogonal based on the inner product $\langle\cdot, \cdot\rangle_{i+1, c}$. Denote the discrete norm defined by the inner product $\langle\cdot, \cdot\rangle_{i+1, c}$ as $\|\cdot\|_{i+1, c}$, that is $\|h\|_{i+1, c}^{2}=\boldsymbol{v}^{\mathrm{T}} \boldsymbol{H}_{i+1}^{(c)-1} \boldsymbol{v}$ for $h=\boldsymbol{N}_{i+1, k} \boldsymbol{v} \in \boldsymbol{V}_{i+1}$. It is not difficult to prove the following theorem.

Theorem 1 For a B-spline function $f_{i+1}=\boldsymbol{N}_{i+1, k} \boldsymbol{d}_{i+1} \in \boldsymbol{V}_{i+1}$, suppose that the lower resolution part of $f_{i+1}$ is $f_{i}=\boldsymbol{N}_{i, k} \boldsymbol{d}_{i} \in \boldsymbol{V}_{i}$

$$
\left\|f_{i}-f_{i+1}\right\|_{i+1, c}=\min \left\{\left\|h_{i}-f_{i+1}\right\|_{i+1, c} \mid h_{i} \in \boldsymbol{V}_{i}\right\}
$$

Theorem 1 show that the lower resolution part $f_{i}$ of $f_{i+1} \in \boldsymbol{V}_{i+1}$ decomposed by new wavelets is the orthogonal projection of $f_{i+1}$ into lower resolution scale space $\boldsymbol{V}_{\boldsymbol{i}}$ based on the discrete inner product $\langle\cdot, \cdot\rangle_{i+1, c}$.

Let $\boldsymbol{v}=\left[v_{0} v_{1} \cdots v_{n_{i+1}}\right]^{\mathrm{T}}$. By simple computation we have

$$
\begin{aligned}
\boldsymbol{v}^{\mathrm{T}} \boldsymbol{H}_{i+1, t, t^{\prime}}^{(c)-1} \boldsymbol{v}= & v_{0}^{2}+\cdots+v_{t+u}^{2}+\left(v_{t+u+1}-c v_{t^{\prime}-u-1}\right)^{2}+\left(v_{t+u+2}-c v_{t^{\prime}-u-2}\right)^{2} \\
& +\cdots+\left(v_{t^{\prime}}-c v_{t}\right)^{2}+v_{t^{\prime}+1}^{2}+\cdots+v_{n_{i+1}}^{2}
\end{aligned}
$$

where

$$
u=\left\lceil\left(t^{\prime}-t-1\right) / 2\right\rceil .
$$

$(1-|c|) \boldsymbol{v}^{\mathrm{T}} \boldsymbol{v} \leq \boldsymbol{v}^{\mathrm{T}} \boldsymbol{H}_{\left.i+1, t, t^{(}\right)}^{(c)-1} \boldsymbol{v} \leq(1+2|c|) \boldsymbol{v}^{\mathrm{T}} \boldsymbol{v}$ if $c<1$. Note that in the Equation $\quad \boldsymbol{H}_{i+1}^{(c)-1}=\prod_{i=1}^{r} \boldsymbol{H}_{i+1, \gamma_{i+1}\left(j_{s}\right), \beta_{i+1}\left(j_{s}\right)}^{(c)-} \quad$ we always have $\beta_{i+1}\left(j_{s}\right)<\gamma_{i+1}\left(j_{s+1}\right)$. Then from Eq. (7) we have

$$
(1-|c|)\|v\|_{l_{2}} \leq\|v\|_{i+1, c} \leq(1+2|c|)\|v\|_{l_{2}},
$$

where $c<1$. Eq. (8) reveals an equivalent relation between discrete norms $\|\cdot\|_{i+1, c}$ and $\|\cdot\|_{l_{2}}$. It implies that new wavelets possess the same good approximation property as that of Pan wavelets.

\section{CONCLUTION}

In the paper a kind of biorthogonal nonuniform B-spline wavelets is constructed. From the view of application, proposed wavelets possess the smallest supports. In additional to having small supports, the proposed wavelets are simple and efficient in computation, possess good approximation property and are flexible in the application for the multiresolution modeling of NURBS curves and surfaces.

\section{ACKNOWLEDGMENT}

This work is supported by Nature Science Foundation of Fujian province, China (No. 2010J01318).

\section{REFERENCES}

[1] Finkelstein A.; Salesin, D. H.. Multiresolution Curves. In: ACM Siggraph'94 [C], 1994, PP: 261-268.

[2] Lyche T.; M $\Phi$ rken K.; Quak E.. Theory and Algorithms for Nonuniform Spline Wavelets. In: Multivariate Approximation and Applications [M], Cambridge University press, Cambridge, 2001, PP:152-187.

[3] LI Deng-gao; QIN Kai-huai; SUN Han-qiu.. Curve Modeling with Constrained B-spline Wavelets. Computer Aided Geometric Design [J], 2005, 22 (1), PP:45-56.

[4] Bertram M.. Single-knot Wavelets for Non-uniform B-splines. Computer Aided Geometric Design [J], 2005, 22 (9), PP 849-864.

[5] PAN Ri-jing; YAO Zhi-qiang. Biorthoganal Nonuniform B-spline Wavelets Based on a Discrete Norm. Computer Aided Geometric Design [J], 2009, 26 (4), PP: 480-492.

[6] Cohen E.; Lyche T.; Riesenfeld R.. Discrete B-splines and Subdivision Techniques in Computer- aided Geometric Design and Computer Graphics. Computer Graphics and Image Processing [J], 1980, 14 (2), PP:87-111.

[7] PAN Ri-jing; WENG Bin. Recursive Representation and Application of Transformation Matrices of B-spline Bases. Computer Aided Geometric Design [J], 2009, 26 (1), PP:82-93. 